Journal of Case Reports 2020;10(2):72-74

\title{
Isolated Solitary Metachronus Perirenal Metastasis in a Distal Gastric Cancer
}

\author{
Deepak Prakash Bhirud, Sunil Kumar, Shiv Charan Navriya, Satish Kumar Ranjan, Kim Jacob Mammen \\ Department of Urology, All India Institute of Medical Sciences, Rishikesh, Uttarakhand, India.
}

\section{Corresponding Author:}

Dr Sunil Kumar

Email: chaurasiasunilbdev@gmail.com

This is an Open Access article distributed under the terms of the Creative Commons Attribution License (creativecommons.org/ licenses/by/3.0).

Received : November 8, 2019

Accepted : March 30, 2020

Published : April 10,2020

\begin{abstract}
Background: Perirenal metastasis in gastric adenocarcinoma is rarely reported. We report an isolated solitary perirenal metastasis from gastric adenocarcinoma in a metachronus setting. Case Report: A 46 year old male patient, with a history of carcinoma stomach, and distal gastrectomy and adjuvant chemo-radiotherapy, came for regular follow up. His four year follow up CT scan showed a $3 \times 3 \times 3 \mathrm{~cm}$ mass in right perirenal space and it was FDG avid on PET CT. He underwent robot assisted laparoscopic excision of mass. It was adenocarcinoma suggestive of metastasis. Patient responded to 6 cycles of chemotherapy after metastectomy. This is the first case of isolated metachronus perirenal metastasis from adenocarcinoma of stomach. Conclusion: Gastric malignancy requires stringent follow up for timely detection of any metachronous metastasis and its appropriate treatment.
\end{abstract}

Keywords: Adenocarcinoma, Chemotherapy, Gastrectomy, Laparoscopy, Robotics, Stomach Neoplasms.

\section{Introduction}

The most common site of metastasis from gastric malignancy is liver. An isolated metastasis to perirenal space from gastric adenocarcinoma in metachronous setting is very rare. If extensively involved, it may result in nephrectomy during metastectomy or may be unresectable. We hereby present a case of isolated metachronous perirenal metastasis following successful management of gastric adenocarcinoma. Robot assisted metastectomy was done and patient is asymptomatic presently.

\section{Case Report}

A 46 year old male initially presented with history of abdominal distension and bloating. On evaluation, UGI endoscopy showed large ulcer involving the incisura, antrum and pylorus [Fig. 1a]. CECT confirmed the same with heterogeneously enhancing wall thickening of $1.25 \mathrm{~cm}$ involving incisura till first part of duodenum. Surrounding fat planes were maintained and there was no locoregional lymphadenopathy. Biopsy was suggestive of gastric adenocarcinoma. He underwent distal gastrectomy, omentectomy with antecolic gastrojejunostomy. No lymphadenectomy was performed because of absence of lymphadenopathy on cross sectional images. Histopathology report showed adenocarcinoma stomach (diffuse gastric typepT3NxMx) and omentum was free of tumor. Patient received concurrent chemotherapy/radiotherapy (5-FU and 25 \# fraction 45 Gy to tumor bed).

Patient was kept on regular follow up as per treatment guidelines. Four years later during follow up, patient developed a solitary $3 \times 3 \times 3 \mathrm{~cm}$ right perinephric metastatic deposit on CECT abdomen [Fig.1b] which was confirmed by PET CT [Fig.1c,d]. Patient underwent a robot assisted laparoscopic metastatectomy [Fig.2a,b]. Histopathological examination showed metastatic deposits of adenocarcinoma [Fig.3]. Patient received $1^{\text {st }}$ cycle of $\mathrm{mTPF}$ (modified 
docetaxel, cisplatin, and fluorouracil) according to initial histopathology report of metastectomy. Subsequent immunohistochemistry

(IHC) showed strong positivity for CK-7 [Fig.3a], weak positivity for CK-20 [Fig.3c] suggesting gastric adenocarcinoma. Her2neu was also strongly positive [Fig.3d]. Therefore chemotherapy was switched to transtuzumab, cisplatin, capecitabine. Patient received 6 cycles of chemotherapy and tolerated well.

\section{Discussion}

Perirenal space is a part of retroperitoneal space formed of anterior (Gerota) and posterior (Zuckerkandl) renal fascia. These fascia fuses laterally to form lateroconal fascia. Superiorly they are fixed to diaphragmatic fascia above the adrenals. Perirenal space is divided into multiple compartments by thin, fibrous septa called as Kunin septa. Both perirenal spaces communicate with each other in midline and pelvic retroperitoneal space below [1].

Perirenal space harbours variety of pathology including benign and malignant. Primary tumors of perirenal space are rare. Isolated perirenal lesions are not common. Castleman disease, perirenal hemangiona, perirenal lymphangioma and extra-intestinal stromal tumor sometimes have isolated primary focal lesions to name a few [2]. Isolated metastasis in perirenal space is rare. Metastasis to perirenal space is predominantly from lung, malignant melanoma, breast, prostate carcinoma and gastrointestinal tract. Lung cancer have predilection for perirenal space secondary to cross connections between mediastinal and perirenal lymphatic vessels $[1,3]$. Malignant melanoma, breast, prostate carcinoma and gastrointestinal tract malignancy spread to perirenal space by hematogenous route. Frequently perirenal metastasis are multiple and bilateral [4]. La Fianza A et al. published largest case series of nine cases of isolated perirenal metastasis in which only four were solitary. Only two cases

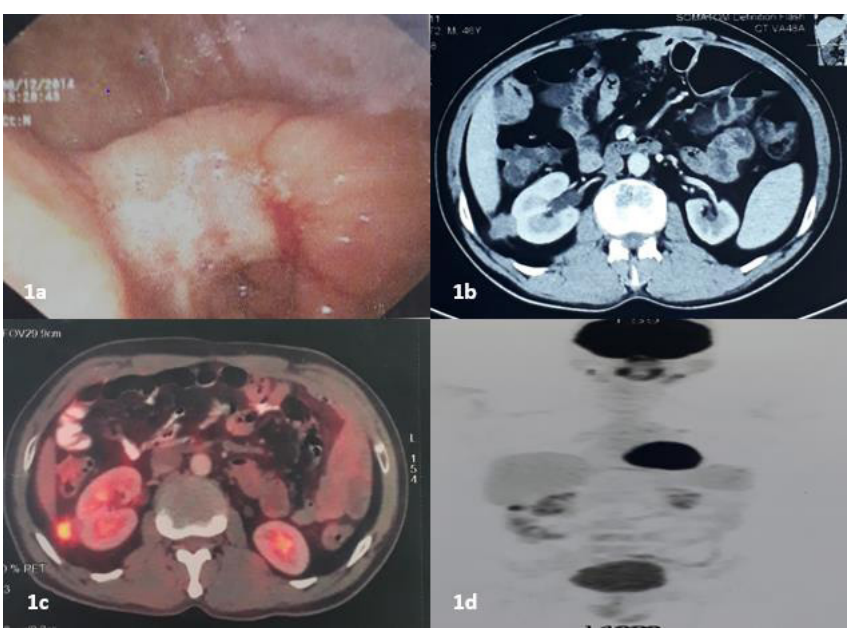

Fig.1 (a): UGIE showing large ulcer involving incisura, antrum and pylorus; (b): CECT abdomen showing perianal metastasis; (c,d): PET CT FDG avid lesion in perirenal space.

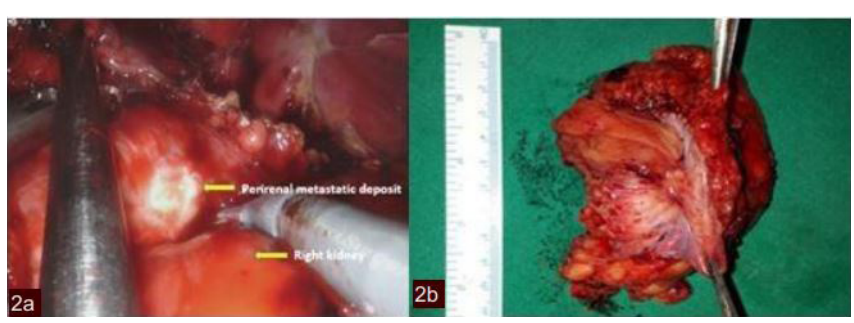

Fig.2 (a): Intra-opearative image; (b): specimen.

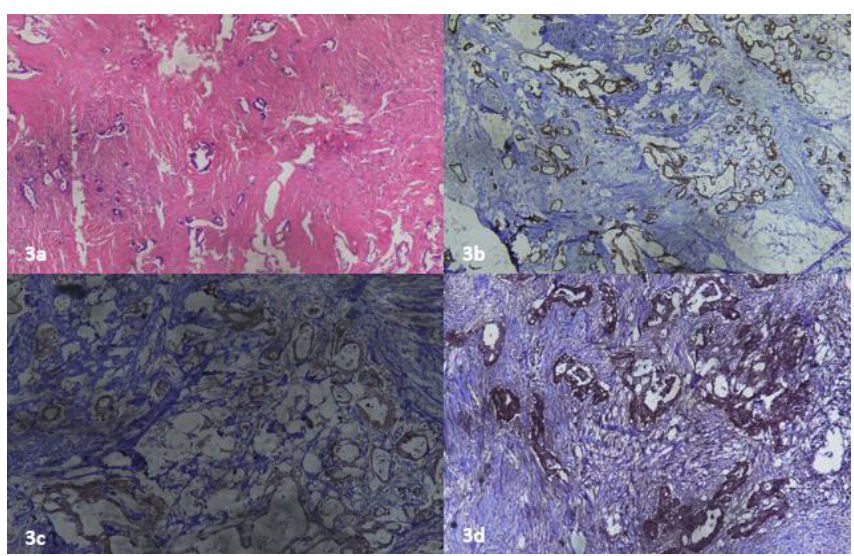

Fig.3 (a): Adenocarcinoma; (b): IHC strong positivity for CK 7; (c): IHC weak positivity for CK 20; (d): IHC strong positivity for Her2neu.

were from gastrointestinal tract. Two cases were detected at pathological autopsy [5]. Their study also highlighted the poor prognosis in patients with isolated perirenal metastasis. Gastric malignancies 
have different histology. Diffuse gastric type is one of them and have bad prognosis. The most common sites of metastasis from gastric cancer are liver, peritoneum, lung and bone [6].

Our patient had isolated, solitary, metachronus metastasis from distal gastric adenocarcinoma. Patient was tumor free for four and half years after initial treatment. There is no reported large series on isolated perirenal metastasis and their prognosis in these patients. This case highlights the metastatic behaviour of gastric malignancy.

\section{Conclusion}

Isolated, solitary perirenal space metastatic mass in distal gastric cancer is a rare entity and can be managed with metastectomy with minimally invasive approach. Regular follow up is needed.

Contributors: DPB conceived the presented idea, and revised the manuscript in consultation with SK. SCN, SKR, and KJM read and provided critical feedback for the manuscript. SK will act as the study guarantor. All authors approved the final version of the manuscript and are accountable for all aspects of the study in ensuring its accuracy and integrity.

Funding: None; Competing interests: None stated.

\section{References}

1. Gore RM, Balfe DM, Aizenstein RI, Silverman PM. The great escape: interfascial decompression planes of the retroperitoneum. AJR Am J Roentgenol. 2000;175:363370.

2. Surbhi VR, Menia C, Prasad SR, Patel AH, Nagar A, DalrympleNC.Neoplastic and nonneoplastic proliferative disorders of the perirenal space: cross sectional imaging findings. Radiographics. 2018;28:1005-1017.

3. Wilbur AC, Turk JN, Capek V. Perirenal metastases from lung cancer: CT diagnosis. J Comput Assist Tomogr. 1992;16:589-591.

4. Grutzner G, Jungblut RM. Perirenal metastasis of a malignant melanoma in a young child. Aktuelle Radiol. 1993;3:372-374.

5. La Fianza A, Maccabelli G, Van Der Byl G, Prevedoni Gorone MS, Gregoli B. Isolated nodular metastases in perirenal space: our experience of nine cases and review of the literature. Minerva Urol Nefrol. 2011;63:281-286.

6. Riihimäki M, Hemminki A, Sundquist K, Sundquist J, Hemminki K. Metastatic spread in patients with gastric cancer. Oncotarget. 2016;7:52307-16. 